\title{
Viable super inflation scenario from $F(T)$ modified teleparallel gravity
}

\author{
A. I. Keskin ${ }^{\text {a }}$ \\ Department of Energy System Engineering, Sirnak University, Şirnak, Turkey
}

Received: 29 July 2018 / Accepted: 28 August 2018 / Published online: 3 September 2018

(C) The Author(s) 2018

\begin{abstract}
Viability of the super inflation scenario is discussed in $F(T)$ modified teleparallel gravity. We calculate the power spectrum of the primordial curvature and tensor perturbations, and we derive two important parameters corresponding to these perturbations, i.e., spectral index parameter $n_{s}$ and scalar-to-tensor ratio $r$. We observe that the super inflation scenario provides a nearly scale invariant power spectrum of scalar and tensor perturbations in the observational bounds of $n_{s}$ and $r$, according to choosing the proper values interval of the power-term of the scale factor, $a \sim t^{h}$.
\end{abstract}

\section{Introduction}

The inflation scenario [1-3], which is supported by observational data [4], is appeared as a successful mechanism which describes the the early-time evolution of the universe. This scenario working at the high energy densities of the universe is generally described by a scalar field which is minimal coupled with gravity, whose potential function is nearly a flat form. When the scalar field slowly exhibits a motion on the flat-potential hill, the universe inflates exponentially, which this results an accelerated expansion case (quasi-de Sitter expansion), named as slow-rolling mechanism. In this mechanism, the accelerated expansion case is provided as long as the slow-roll parameter, $\epsilon=\frac{\dot{H}}{H^{2}}$, is smaller than one [5]. However, the large-structure of the universe is connected to beginning evolution of the universe, as the inflation scenario predicts quantum perturbations which plant the seeds on spacetime tissue. The traces of this primordial fluctuations are observed in the cosmic microwave background (CMB) which spreads freely in the universe. Hence, testing the inflation scenarios are made by measuring $\mathrm{CMB}$ anisotropies and by calculating useful two parameters coming from the two types of perturbations [6] which are related to metric perturbations: as mentioned above, the first is the scalar (curva-

a e-mails: alikeskin039@gmail.com; a.ihsankeskin@ sirnak.edu.tr ture) perturbations, $P_{R}$, take on a task as producing the seeds which form the present universe, and the second is the tensor perturbations, $P_{T}$, which are dubbed as gravitational waves of the early-universe. In the literature, under the slow-roll condition $\epsilon \ll 1$ it was developed many different inflation scenarios such as the chaotic inflation [7], the quintessential inflation [8], k-inflation [9], the Brane inflation [10,11], the warm inflation [12-14], the intermediate inflation [15], the super inflation [16] and so on.

In this study, we examine the super inflation scenario in the context of $F(T)$ gravity theory. This gravity theory, which is formed by tetrad fields $e_{\mu}^{A}$ [17] instead of metric tensor $g^{\mu \nu}$, was developed as an alternative theory to the standard Einstein's gravity. In the fundamental idea of teleparallelism, there is only a torsion $T$ corresponding to Weitzenböck connection which is formed by dynamical objects $e_{\mu}^{A}$ of the gravity theory. A generalized version of the torsion gravity is $F(T)$ gravity theory constructed by [18], where the functional form of $T$ is appeared in the action integral (which is similar to the structure of the $F(R)$ gravity in the EinsteinHilbert action). In the context of $F(T)$ gravity, late-time cosmic acceleration (dark energy) of the universe was discussed in several studies [19-28], and particular cosmological solutions and applications can be found in Refs. [29,30]. The studies on the intermediate inflation scenario in the context of this gravity theory were performed in Refs. [31-35]. Cosmological perturbations for inflationary scenarios were studied in Refs. [36-39], and also some examinations on inflation of the universe in the context of $F(T)$ gravity can be seen in Refs. [40-42] . However, investigations of thermodynamical perspective of the gravity theory were discussed in Refs. [43-45].

In this study, we show the super inflation scenario in the framework of $F(T)$ modified teleparallel gravity. Using a notation $A$ [46] we re-construct an energy equation (friedmann eq.), and we realize a unified solution $[47,48]$ of it. However, using a notation $M$ which is similar to $A$, we re- 
construct the perturbation equations of $F(T)$ gravity. After that we calculate the power spectrum of the primordial curvature and tensor perturbations, and two important parameters corresponding to these perturbations, i.e., spectral index parameter $n_{s}$ and scalar-to-tensor ratio $r$. We observe that the super inflation scenario provides a nearly scale invariant power spectrum of scalar and tensor perturbations in the observational bounds of $n_{s}$ and $r$ [4].

\section{$2 F(T)$ modified teleparallel gravity}

The action integral of $F(T)$ gravity is given by

$$
S=\int d^{4} x|e|\left[\frac{F(T)}{2 \kappa^{2}}+L_{m}\right]
$$

Variation of the action with respect to tetrad field gives the following equation,

$$
\begin{aligned}
& e^{-1} \partial_{\mu}\left(e S_{A}^{\mu \nu}\right) F_{T}-e_{A}^{\lambda} T_{\mu \lambda}^{\rho} S_{\rho}^{\nu \mu} F_{T}+S_{A}^{\mu \nu} \partial_{\mu} T F_{T T} \\
& +\frac{1}{4} e_{A}^{v} F=\frac{\kappa^{2}}{2} e_{A}^{\rho} T_{\rho}^{\nu},
\end{aligned}
$$

where $F_{T}$ is derivative with respect to the torsion scalar. In this study, we shall use the notations in Ref. [37]. Hence, indices $A$ shows the numbers $0,1,2,3$ on the tangent spacetime, and metric tensor $g^{\mu v}$ indicates the relation $g_{\mu \nu}=$ $\eta_{A B} e_{\mu}^{A} e_{v}^{B}$ in which all coordinate space-time are transported on the tangent spacetime manifold via tetrad fields $e_{\mu}^{A}$. The torsion $T_{\mu \nu}^{\rho}$ and the contorsion tensors are defined as [17]

$$
\begin{aligned}
& T_{\mu \nu}^{\lambda}=e_{A}^{\lambda}\left(\partial_{\mu} e_{\nu}^{A}-\partial_{\nu} e_{\mu}^{A}\right), \\
& K_{\rho}^{\mu \nu}=-\frac{1}{2}\left(T_{\rho}^{\mu \nu}-T_{\rho}^{\nu \mu}-T_{\rho}^{\mu \nu}\right) .
\end{aligned}
$$

Also, the torsion scalar $T$ is defined by

$T \equiv S_{\rho}^{\mu \nu} T_{\mu \nu}^{\rho}$

with $S_{\rho}^{\mu \nu}=\frac{1}{2}\left(K_{\rho}^{\mu v}{ }_{\rho}+\delta_{\rho}^{\mu} T_{\alpha}^{\alpha \nu}-\delta_{\rho}^{v} T_{\alpha \mu}^{\alpha \mu}\right)$. In the background of the following metric function,

$d s^{2}=-d t^{2}+a^{2}(t)\left[d x^{2}+d y^{2}+d z^{2}\right]$,

the following friedmann equations can be derived from Eq. (2),

$$
\begin{aligned}
3 H^{2} & =\kappa^{2}\left(\rho_{m}+\rho_{d}\right)=\kappa^{2} \rho_{e}, \\
-\left(2 \dot{H}+3 H^{2}\right) & =\kappa^{2}\left(p_{m}+p_{d}\right)=\kappa^{2} p_{e} .
\end{aligned}
$$

where

$$
\begin{aligned}
\rho_{d}= & \frac{1}{2 \kappa^{2}}\left(-T-F+2 T F_{T}\right), \\
p_{d}= & \frac{1}{2 \kappa^{2}}\left(-4 \dot{H}+4 \dot{H} F_{T}\right. \\
& \left.\quad+8 \dot{H} T F_{T T}+T+F-2 T F_{T}\right) .
\end{aligned}
$$

Herein, $T=-6 H^{2}$. For a general expression, Friedmann Eqs. (6), (7) can be re-constructed by using the following notation[16]

$A=8 \pi G\left(p_{e}-\rho_{e} w\right)+2 \dot{H}+3 H^{2}(1+w)$,

then we can write the following energy equation

$$
\begin{aligned}
& 2 \dot{H} F_{T}+4 \dot{H} T F_{T T} \\
& \left.+\frac{(1+w)}{2}\left(T+F-2 T F_{T}\right)\right)+3 H^{2}(1+w)=A
\end{aligned}
$$

where $A=0$, with the defination of the effective parameter $w_{e}=-1-\frac{2 \dot{H}}{3 H^{2}}$ and $w=w_{e}$. The Eq. (11) has a secondorder structure and can be used to describe evolution of the early inflationary universe because it includes the Hubble parameter and its derivatives and also the effective parameter.

\subsection{Perturbation equations}

Scalar-type metric fluctuations are descibed by the following perturbed metric function:

$d s^{2}=(1+2 \Phi) d t^{2}-a(t)(1-2 \Psi) \sum d x_{i}^{2}$,

where $\Phi$ and $\Psi$ are two scalar functions which characterize scalar metric fluctuations. In this case, the torsion scalar can be expressed in terms of these functions as

$\delta T=12 H(\dot{\Phi}+H \Psi)$.

According to perturbed metric (12), perturbation equations corresponding to

$F(T)=T+f(T)$ 
gravity are obtained as $[31,37]$

$$
\begin{aligned}
& \left(1+f_{T}\right) \frac{\nabla^{2} \Psi}{a^{2}}-3\left(1+f_{T}\right) H \dot{\Psi} \\
& \quad-3\left(1+f_{T}\right) H^{2} \Phi+36 f_{T T} H^{3}(\dot{\Psi}+H \Phi) \\
& \quad=4 \pi G \delta \rho, \\
& \left(1+f_{T}-12 H^{2} f_{T T}\right)(\dot{\Psi}+H \Phi)=4 \pi G \delta q, \\
& \left(1+f_{T}\right)(\Psi-\Phi)=8 \pi G \delta s, \\
& \left(1+f_{T}-12 H^{2} f_{T T}\right) \ddot{\Psi}+3 H\left(1+f_{T}-12 H^{2} f_{T T}\right. \\
& \left.\quad-12 \dot{H} f_{T T}+48 H^{2} \dot{H} f_{T T T}\right) \dot{\Psi}+\left[3 H^{2}\right. \\
& \quad \times\left(1+f_{T}-12 H^{2} f_{T T}\right)+2 \dot{H}\left(1+f_{T}-30 H^{2} f_{T T}\right. \\
& \left.\left.+72 H^{4} f_{T T T}\right)\right] \Phi+\frac{1+f_{T}}{2 a^{2}} \nabla^{2}(\Psi-\Phi)=4 \pi G \delta p .
\end{aligned}
$$

Herein, functions $\delta \rho, \delta p, \delta q$ and $\delta s$ show the fluctuations of energy density, pressure, fluid velocity, and anisotropic stress, respectively. We assume that the matter component is in the form of canonical scalar field which has a potential $V(\phi)$. In this case the equation of motion for scalar field is as follows,

$\ddot{\phi}+3 H \dot{\phi}+V_{\phi}=0$.

Then, for perturbed quantities we can write [37]

$$
\begin{aligned}
\delta \rho & =\dot{\phi}(\delta \dot{\phi}-\dot{\phi} \Phi)+V_{\phi} \delta \phi, \\
\delta q & =\dot{\phi} \delta \phi, \\
\delta s & =0, \\
\delta p & =\dot{\phi}(\delta \dot{\phi}-\dot{\phi} \Phi)-V_{\phi} \delta \phi .
\end{aligned}
$$

The condition (22) in (17) implies that $\Phi=\Psi$. Hence, the complete equation of scalar perturbations is determined by gravitational potential $\Phi$. To write perturbed equation in a unified form we shall use the following notation,

$M=(\delta p-w \delta \rho) 8 \pi G$,

which is similar to the notation (10), where $\kappa^{2}=8 \pi G$. Then we find

$$
\ddot{\Phi}_{k}+\alpha \dot{\Phi}_{k}+\mu^{2} \Phi_{k}+c_{s}^{2} \frac{k^{2}}{a^{2}} \Phi_{k}=0
$$

Herein, $\Phi_{k}$ is one Fourier mode of the potential $\Phi$ and $k$ is the comoving wave vectors [49]. Also, $\alpha, \mu^{2}$ and $c_{s}^{2}$ denote the frictional term, the effective mass and the speed of sound parameter, respectively, given by

$$
\begin{aligned}
\alpha & =7 H+\frac{2 V_{\phi} \dot{\phi}-\frac{36 H \dot{H}\left(f_{T T}-4 H^{2} f_{T T T}\right)}{1+f_{T}-12 H^{2} f_{T T}},}{\mu^{2}}=6 H^{2}+2 \dot{H}+\frac{2 V_{\phi} H}{\dot{\phi}}-\frac{36 H^{2} \dot{H}\left(f_{T T}-4 H^{2} f_{T T T}\right)}{1+f_{T}-12 H^{2} f_{T T}}, \\
c_{s}^{2} & =\frac{-w\left(1+f_{T}\right)}{1+f_{T}-12 H^{2} f_{T T}} .
\end{aligned}
$$

Thus, using energy Eq. (6) the master Eq. (11) can be written as

$\dot{H} f_{T}-12 \dot{H} H^{2} f_{T T}=-4 \pi G\left(\rho_{\phi}+p_{\phi}\right)=-4 \pi G \dot{\phi}^{2}$,

where the pressure $p_{\phi}=\frac{\dot{\phi}^{2}}{2}-V(\phi)$ and energy density $\rho_{\phi}=$ $\frac{\dot{\phi}^{2}}{2}+V(\phi)$ of the scalar field show the following continuity equation

$\dot{\rho}_{\phi}+3 H\left(\rho_{\phi}+p_{\phi}\right)=0$.

In order to write the perturbation Eq. (25) in a simple form, we use time derivative of Eq. (27), which gives the following expression,

$$
\frac{36 H\left(f_{T T}-4 H^{2} f_{T T T}\right)}{f_{T}-12 H^{2} f_{T T}}=\frac{6 H}{\dot{H}}+\frac{2 V_{\phi}}{\dot{H} \dot{\phi}}+\frac{\ddot{H}}{\dot{H}^{2}},
$$

where Eq. (19) is used. We now proceed by finding the form of $f(T)$ gravity to write the Eq. (29) in a simple frame. For this, we use master Eq. (27), which can be written as follows

$$
2 T^{2} f_{T T}+T f_{T}=-\frac{\kappa^{2} \rho_{0}\left(1+w_{\phi}\right) 3 h}{\left(-6 h^{2}\right)^{\frac{3 h\left(1+w_{\phi}\right)}{2}} T^{\frac{3 h\left(1+w_{\phi}\right)}{2}}}-T,
$$

where the power law solutions of the scale factor are used,

$a \sim t^{h}, \quad t=\left(-6 h^{2}\right)^{\frac{1}{2}} T^{-\frac{1}{2}}$.

Also, $w_{\phi}=\frac{p_{\phi}}{\rho_{\phi}}$ is equation-of-state (EoS) parameter for scalar field and $\rho_{0}$ is an integral constant coming from the solution of Eq. (28). The solution of differential Eq. (30) is

$$
\begin{gathered}
f(T)=- \\
T-\frac{6^{-\frac{3 h\left(1+w_{\phi}\right)}{2}} \kappa^{2} \rho_{0}}{2^{-1}\left(-h^{2}\right)^{\frac{3 h\left(1+w_{\phi}\right)}{2}}\left(-1+3 h\left(1+w_{\phi}\right)\right)} \\
T^{\frac{3 h\left(1+w_{\phi}\right)}{2}}+2 \sqrt{T} c_{1}+c_{2} .
\end{gathered}
$$

Due to the metric (5) showing spatial flat-FRW universe model, to obtain real value of the function (32) we use standart FRW solutions, $\kappa^{2} \rho_{0}=3 h^{2}$ and $h=\frac{2}{3\left(1+w_{\phi}\right)}$. Then we find $f(T)=0$ with integral constants $c_{1}=c_{2}=0$. Hence, $F(T)$ gravity given by (14) is read as $F(T)=T$. And inserting $F(T)=T$ into (29) we obtain

$$
\left(3 H+\frac{\ddot{H}}{2 \dot{H}}\right) \dot{\phi}=-V_{\phi} .
$$


Thus, the expressions in (26) can be written as follows,

$$
\begin{aligned}
& \alpha=H-\frac{\ddot{H}}{\dot{H}}, \\
& \mu^{2}=2 \dot{H}-\frac{\ddot{H} H}{\dot{H}}, \\
& c_{s}^{2}=-w_{\phi} .
\end{aligned}
$$

As a result, perturbation Eq. (25) is converted into the perturbation equation in the standard Einstein gravity [50] if $w_{\phi}=0$,

$$
\begin{aligned}
& \ddot{\Phi}_{k}+\left[H-\frac{\ddot{H}}{\dot{H}}\right] \dot{\Phi}_{k} \\
& +\left[2 \dot{H}-\frac{\ddot{H} H}{\dot{H}}\right] \Phi_{k}-w_{\phi} \frac{k^{2}}{a^{2}} \Phi_{k}=0 .
\end{aligned}
$$

It is know that the speed of sound parameter takes the values $0 \leq c_{s}^{2} \leq 1$ interval which provides that $-1 \leq w_{\phi} \leq 0$. This range is similar to the range of EoS parameter of a tachyon scalar field [51,52] which can be written for the metric (5). Hence, the nature of this parameter can produce an acceleration. Therefore, with the standard Einstein approximation $F(T)=T$ we shall show that the super inflation scenario produces a nearly scale invariant power spectrum compatible with observations, as perturbation Eq. (35) is governed by last term which includes a tachyon-like EoS parameter. Therefore, the last term is an important point of the present study which separates it from the standard Einstein-FRW cosmology.

Due to $F(T)=T$ cosmology, to characterize the perturbation Eq. (35) we make use of a gauge-invariant variable $\zeta$ which is defined in the standard cosmological perturbation theory,

$\zeta=\Phi-\frac{H}{\dot{H}}(\dot{\Phi}+H \Phi)$.

From the time derivatives of $\zeta$ we can write

$$
\begin{aligned}
& \zeta^{\prime}=-\frac{w_{\phi} k^{2} \Phi}{\epsilon a H}, \\
& \zeta^{\prime \prime}=w_{\phi} k^{2} \zeta-2 \zeta^{\prime} \dot{a},
\end{aligned}
$$

where "prime" shows differentiation with respect to the conformal time which is defined as follows

$\tau=\int \frac{d t}{a}$.

However, we define a new variable as

$v_{k}=z \zeta$, where

$z=a \theta$.

Also, $\theta$ is a real constant parameter. As a result, equation of motion for each mode function $v_{k}$ is obtained as

$v_{k}^{\prime \prime}+\left(-w_{\phi} k^{2}-\frac{z^{\prime \prime}}{z}\right) v_{k}=0$.

Using the solution (31) we can re-write Eq. (41) as follows

$v_{k}^{\prime \prime}+\left[-w_{\phi} k^{2}-\frac{h(2 h-1)}{(1-h)^{2} \tau^{2}}\right] v_{k}=0$.

\subsection{Viability of super inflation scenario}

The super inflation mechanism predicts the three phase regions for the early universe. The first is a vacuum state $w_{\phi} \sim-1$. The second is a quintessential region $-1<$ $w_{\phi}<-\frac{1}{3}$, with super accelerated solution $h=\frac{4}{3\left(1+w_{\phi}\right)}$, where $h>2$. Then the matter creation region is followed $-\frac{1}{3}<w_{\phi}<\frac{1}{3}$, where $1<h<2$. We examine perturbation Eq. (42) of $F(T)$ cosmology for these regions.

\subsubsection{Vacuum state and quintessential expansion}

In a vacuum-like case (quasi-de Sitter case) $w_{\phi} \sim-1$, and so at the limit $h=\frac{4}{3\left(1+w_{\phi}\right)} \rightarrow \infty$, Eq. (42) is reduced to the form,

$v_{k}^{\prime \prime}+\left[k^{2}-\frac{2}{\tau^{2}}\right] v_{k}=0$,

which is known as the Mukhanov-Sasaki equation. At subhorizon scale $\tau \rightarrow-\infty$ or $k \gg a H$, the Eq. (43) is read as a simple oscillator equation $v_{k}^{\prime \prime}+k^{2} v_{k}=0$. The solution is found as [37,53]

$v_{k}(\tau) \simeq \frac{e^{-i k \tau}}{\sqrt{2 k}}$

which is known as the Bunch-Davies vacuum state. This means that the initial condition of the super inflation scenario is a Bunch-Davies vacuum state as a result of fluctuations at quantum zero point energy level. On the other hand, the amplitude of each mode function $v_{k}$ should be determined at horizon crossing $k=a H$. Hence, the solution of Eq. (42) will be as follows,

$v_{k}(\tau)=\sqrt{\tau}\left[C_{1} J_{(A)}\left(-i k \tau \sqrt{w_{\phi}}\right)+C_{2} Y_{(A)}\left(-i k \tau \sqrt{w_{\phi}}\right)\right]$ 
where $A=\frac{3 h-1}{2(h-1)}$, and $J_{(A)}, Y_{(A)}$ show the first and the second kinds of bessel functions, respectively, which is given by

$$
\begin{gathered}
J_{(A)}\left(-i k \tau \sqrt{w_{\phi}}\right)=\frac{1}{\Gamma(A+1)}\left[\frac{-i k \tau \sqrt{w_{\phi}}}{2}\right]^{A}, \\
Y_{(A)}\left(-i k \tau \sqrt{w_{\phi}}\right)=-\frac{\Gamma(A)}{\pi}\left[\frac{2}{-i k \tau \sqrt{w_{\phi}}}\right]^{A} \\
-\frac{\cos (A \pi) \Gamma(-A)}{\pi}\left[\frac{-i k \tau \sqrt{w_{\phi}}}{2}\right]^{A} .
\end{gathered}
$$

As we have discussed in Eq. (35), for the real arguments of functions (47) and (46), the possible values of the EoS parameter should be in $-1 \leq w_{\phi}<0$ interval. Therefore, in this range of the EoS parameter the solution is possible. In other words, the quintessential expansion $-1<w_{\phi}<-\frac{1}{3}$ and dust-like oscillations (matter creation region $w_{\phi} \sim 0$ ) can be observed in the solution above.

Under the approximation $A>0$ and at subhorizon scale $\tau \rightarrow-\infty$ or $t \rightarrow 0^{+}$, the mode function $v_{k}(\tau)$ given by (45) can be written as follows,

$v_{k}(\tau)=\frac{c_{2} \sqrt{\tau}\left(\Gamma(A) 2^{A}\left(-i k \tau \sqrt{w_{\phi}}\right)^{-A}\right.}{\pi}$.

Herein, the constant $c_{2}$ can be determined by comparing (48) with the initial condition (44), which yields to

$c_{2}=\frac{2^{-A} \pi\left(-i k \tau \sqrt{w_{\phi}}\right)^{A}}{\Gamma(A) k^{\frac{1}{2}} 2^{\frac{1}{2}} \tau^{\frac{1}{2}}}$.

The power spectrum of the primordial curvature perturbations should be calculated at $k=a H[31,37,53]$,

$\mathcal{P}_{\zeta}=\frac{k^{3}}{2 \pi^{2}}\left|\frac{v_{k}}{z}\right|_{k=a H}^{2}$.

From (40) the function $z(\tau)$ can be written as follows,

$z(\tau)=(1-h)^{\frac{h}{1-h}} \theta \tau^{\frac{h}{1-h}}$

Using (48) and $z(\tau)$, at horizon crossing $k=a H$, we can write

$\mathcal{P}_{\zeta}=\frac{h^{\frac{2 h}{h-1}}}{4 \pi^{2} \theta^{2}} k^{\frac{2}{1-h}}$.

The power spectrum is not scale invariant as it depends the value $h$. But, in the observational bounds of the spectral index parameter, we can obtain a nearly scale invariant spectrum.
The scalar spectral index parameter, $n_{s}-1=\frac{d \ln \mathcal{P}_{\zeta}}{d \ln k}=$ $\frac{2}{1-h}$, is found as

$n_{s}=\frac{3-h}{1-h}$.

The observational data is constrained the value of the spectral index parameter as follows [4]

$n_{s}=0.965 \pm 0.004$ or $n_{s}=[0.961,0.969]$.

Inserting (53) into (54) we obtain special range of $h$ as follows,

$52.28<h<65.51$

which shows a quintessential expansion interval, $-0.979<$ $w_{\phi}<-0.974$, with the super accelerated solution $h=$ $\frac{4}{3\left(1+w_{\phi}\right)}$. For each certain value of $h$ in the range (55), the power spectrum is almost scale invariant.

\subsubsection{Matter creation region}

After the quintessential expansion, the matter (at least a dustlike matter) should be created from this quintessential type of dark energy. To illustrate this, we shall follow the method proposed by [54] because in our case at hand the left side of the master Eq. (30) can be identified by using the following differential operator

$\widehat{D}=\frac{d}{d t}\left[\frac{\phi}{\ddot{\phi}} \frac{d}{d t}\right]$

This operator produces a similar structure to the left side of master Eq. (30) in terms of cosmic time when applying any function. Because, the scalar field is a power-law form of cosmic time. That is, the solution (33) with (31) is in a form as follows,

$\phi=\left[\frac{n(2-n)}{2(1-3 h)}\right]^{\frac{1}{(2-n)}} t^{\frac{2}{(2-n)}}$

with an integral constant $c=0$. Herein, we assume the chaotic polynomial potential [7],

$V(\phi)=\phi^{n}$

On the other hand, we now take the following notation

$K=\rho_{\phi}\left(w_{\phi}-w_{x}\right)$

which is equal to zero when $w_{\phi}=w_{x}$. In other words, the notation $K$, which is a function of density, is a term that 
is zero when the equation (10) is constructed. Applying the operator (56) to the notation (59) we obtain [54]

$\widehat{D} K=\frac{d}{d t}\left\{\frac{\phi \dot{\phi}}{\ddot{\phi}}\left[\ddot{\phi}\left(1-w_{x}\right)-V_{\phi}\left(1+w_{x}\right)\right]\right\}$

Using Eqs. (28) and (19) we can write

$\widehat{D} \rho_{\phi}\left(1+w_{\phi}\right)=2\left(\dot{\phi}^{2}+\ddot{\phi} \phi\right)$,

where we take into account the friction term $3 H \dot{\phi}$ in Eqs. (19) or (33) for the vacuum state. Then the vacuum case $\dot{\phi}^{2} \ll 1$ and $\ddot{\phi} \ll 1$ in Eq. (61) provides that $w_{\phi} \sim-1$ as we expected. Next, for the quintessential expansion case we can write Eq. (60) as follows,

$\widehat{D} \rho_{\phi}\left(w_{\phi}-w_{x}\right)=2\left(\dot{\phi}^{2}-\phi V_{\phi}\right)$,

where we have neglected the friction term $3 H \dot{\phi}$, that is, the velocity term $\dot{\phi}^{2}$ is dominate in the quintessential region. From zero point condition $w_{\phi} \cong w_{x}$ in which we have obtained the mode function (48) under the condition $\tau \rightarrow$ $-\infty$ which implies a vacuum case for the quintessential region, we have a quantization of the potential as follows,

$\dot{\phi}^{2}=n V(\phi)$.

Then the oscillation EoS parameter of the scalar field can be written as follows,

$w_{\phi}=\frac{n-2}{n+2}$.

Using (64) we now examine dust-like oscillations, which occurs after the quintessential region. For dust-like oscillations $w_{\phi} \sim 0$ corresponding to $n \sim 2$, the perturbation Eq. (42) is as the following

$v_{k}^{\prime \prime}-\frac{20}{\tau^{2}} v_{k}=0$,

In effect, this equation corresponds to the superhorizon scale $\sqrt{-w_{\phi}} k \ll a H$ corresponding to frozen fluctuations. At end of the inflation this means that the freezed modes reenter the horizon at the superhorizon scale, and so the modes of frozen promordial fluctuations are appeared as particles in spacetime tissue at superhorizon (low energy densities) scale.

The solution of Eq. (65) is as the following,

$v_{\kappa}(\tau)=c_{3} \tau^{5}+c_{4} \tau^{-4}$

with the constants $c_{3}, c_{4}$. For end of the inflation $\tau \rightarrow 0^{-}$, the second term $c_{4} \tau^{-4}$ dominates. And the value of $c_{4}$ can be determined by comparing (48) with (66). Then we find $c_{4}=\frac{\tau^{4} k^{\frac{-1}{2}}}{\sqrt{2}}$.

When the same procedure as made in the previous section is followed, we again obtain the equality (53) for the spectral index parameter. This means that the three regions of the super inflation scenario in the context of the perturbation equations of $F(T)=T$ gravity are a single field.

\subsection{Tensor perturbations}

The perturbed FRW metric with tensor perturbation $h_{i j}$, which has properties such as symmetric, transverse, traceless, is given by

$d s^{2}=a(\tau)^{2}\left(-d \tau^{2}+\left(\delta_{i j}+h_{i j} d x^{i} d x^{j}\right)\right)$

Therefore, the tensor $h_{i j}$ has two degrees of freedom. The evolution of the tensor perturbations is described by the following equation $[31,53]$

$\ddot{h}_{i j}+3 H \dot{h}_{i j}-\frac{\nabla^{2}}{a^{2}} h_{i j}=\frac{12 H \dot{H} f_{T T}}{1+f_{T}} \dot{h}_{i j}$.

In our case at hand in which $f(T)=0$, this equation has the following form,

$\ddot{h}_{i j}+3 H \dot{h}_{i j}-\frac{\nabla^{2}}{a^{2}} h_{i j}=0$.

One can expand $h_{i j}$ in Fourier space to calculate the evolution of each tensor Fourier mode $h_{k}(\tau)$,

$h_{i j}(\tau, x)=\int \frac{d^{3} x}{(2 \pi)^{\frac{3}{2}}} h_{k}(\tau, k) e^{i k x}$.

Inserting (71) into Eq. (70) we obtain

$\ddot{h}_{k}+3 H \dot{h}_{k}+\frac{k^{2}}{a^{2}} h_{k}=0$.

However, when the following variable is used,

$v_{k t}=a h_{k}$,

it is found the equation

$v_{k t}^{\prime \prime}+\left(k^{2}-\dot{a}^{2}(2-\epsilon)\right) v_{k t}=0$,

which describes how the perturbation modes are evolve in conformal time. However, in the Eq. (74) the term $\dot{a}^{2}(2-\epsilon)$ 
is equal to $\frac{1}{a} \frac{d^{2} a}{d \tau^{2}}$. Therefore, using the solution $a \sim t^{h}$ we can write Eq. (74) as follows,

$v_{k t}^{\prime \prime}+\left[k^{2}-\frac{h(2 h-1)}{(1-h)^{2} \tau^{2}}\right] v_{k t}=0$,

which is the same with Eq. (42) for $w_{\phi} \sim-1$. Therefore, at subhorizon and at superhorizon scales we find same solutions with Eq. (42). Hence, tensor power spectrum $P_{T}$ matches at horizon crossing $k=a H$, is given by [53]

$P_{T}=\left.\frac{2 H^{2}}{\pi^{2}}\right|_{k=a H}=\frac{2 h^{2} k^{\frac{2}{1-h}}}{h^{\frac{2}{1-h}} \pi^{2}}$,

which is the same with tensor power spectrum defined in the standard Einstein gravity. In our case we have already $F(T)=T$ gravity. Tensor-to-scalar ratio $r=\frac{P_{T}}{P \zeta}$ is found as

$r=8 \theta^{2}$.

Actually, for the possible small values of $\theta$ we can derive observational bounds of the scalar-to-tensor ratio which are given by [4]

$r<0.07$

which corresponds to the range $0<\theta<0.09354$. On the other hand, we can select a proper value of constant parameter $\theta$ to get observational interval (78). For this, when choosing $\theta=\frac{\sqrt{\epsilon}}{2}$ which leads to $r=2 \epsilon$. Then, using super accelerated solution $h=\frac{4}{3\left(1+w_{\phi}\right)}$, with the definition of the EoS parameter, $w_{e}=w_{\phi}=-1+\frac{2 \epsilon}{3}$, we can write the spectral index parameter (53) as follows,

$n_{s}=\frac{4-3 r}{4-r}$

and from which we obtain

$0.061<r<0.076$

together with observational constraint of the spectral index parameter given by (54). This range of the scalar-to tensor ratio is compatible with the Planck data given by (78).

\section{Conclusion}

In this study, we have examined the super inflation scenario in the context of $F(T)$ cosmology. We have used the notation $A$ to construct friedmann equation, and we realize a unified solution of it. Herein, although this solution produces standard Einstein term, i.e., $F(T)=T$, we obtain inflationary regime of the universe with this form of $F(T)$, where the vacuum state and the quintessential region have been obtained at subhorizon scale (high energy densities), and matter creation region is shown at superhorizon scale (low energy densities). On the other hand, we have used the notation $M$ which is structurally similar to $A$ (in which we have followed a method which we refer to equation symmetry), and in this way we obtain scalar perturbation Eq. (35). Viability of the super inflation mechanism is discussed in this equation. We observe that the super inflation scenario produces an amplitude of power spectrum of scalar perturbation which is a nearly scale invariant both at subhorizon and at superhorizon scales, according to special values of $h$ as shown in (55). On the other hand, in the present study, since left side of our master Eq. (30) structurally can be constructed by differential operator (56) in terms of cosmic time, with the scalar field (57), we generally show all regions of the super inflation scenario by using this operator and notation (59) which has the lowest possible energy field when $w_{\phi} \simeq w_{x}$. On the other side, after calculating power spectrum of the tensor perturbation, by taking a proper value of the constant parameter, $\theta=\frac{\sqrt{\epsilon}}{2}$ we obtain the range $0.061<r<0.076$ for the scalar-to-tensor ratio, which this is in good agreement with the Planck data [4].

Open Access This article is distributed under the terms of the Creative Commons Attribution 4.0 International License (http://creativecomm ons.org/licenses/by/4.0/), which permits unrestricted use, distribution, and reproduction in any medium, provided you give appropriate credit to the original author(s) and the source, provide a link to the Creative Commons license, and indicate if changes were made.

Funded by SCOAP ${ }^{3}$.

\section{References}

1. A.H. Guth, Phys. Rev. D 23, 347 (1981)

2. A.D. Linde, Phys. Lett. B 108, 389 (1982)

3. A.A. Starobinsky, Phys. Lett. B 91, 99 (1980)

4. Planck Collab. (N. Aghanim et al.), arXiv:1807.06209 [astroph.CO] (2018)

5. R. Myrzakulov, S.D. Odintsov, L. Sebastiani, Phys. Rev. D 91, 083529 (2015)

6. I. I. Tkachev, arXiv:hep-ph/0405168

7. A.D. Linde, Phys. Lett. B 129, 177 (1983)

8. F. Lucchin, S. Matarrese, Phys. Rev. D 32, 1316 (1985)

9. C. Armendariz-Picon, T. Damour, V.F. Mukhanov, Phys. Lett. B 458, 209 (1999)

10. R. Maartens, D. Wands, B.A. Bassett, I.P.C. Heard, Phys. Rev. D 62, $041301(2000)$

11. T. Golanbari, A. Mohammadi, K. Saaidi, Phys. Rev. D 89, 103529 (2014)

12. A. Berera, Phys. Rev. D 55, 3346 (1997)

13. M. Bastero-Gil, A. Berera, Phys. Rev. D 71, 063515 (2005) 
14. K. Sayar, A. Mohammadi, L. Akhtari, Kh Saaidi, Phys. Rev. D 95, 023501 (2017)

15. J.D. Barrow, P. Saich, Phys. Lett. B 249, 406 (1990)

16. A.I. Keskin, Astrophys. Space Sci. 362, 50 (2017)

17. K. Hayashi, T. Shirafuji, Phys. Rev. D 19, 3524 (1979); Addendumibid. 24, 3312 (1982)

18. G.R. Bengochea, R. Ferraro, Phys. Rev. D 79, 124019 (2009)

19. C.Q. Geng, C.C. Lee, E.N. Saridakis, Y.P. Wu, Phys. Lett. B 704, $384(2011)$

20. E. V. Linder, Phys. Rev. 127301 (2010)

21. J.B. Dent, S. Dutta, E.N. Saridakis, J. Cosmol. Astropart. Phys. 1, 009 (2011)

22. R.-J. Yang, Eur. Phys. J. C 71, 1797 (2011)

23. H. Farajollahi, A. Ravanpak, P. Wu, Astrophys. Space Sci. 338, 23 (2012)

24. V.F. Cardone, N. Radicella, S. Camera, Phys. Rev. D 85, 124007 (2012)

25. K. Bamba, S.D. Odintsov, D. Sez-Gmez, Phys. Rev. D 88, 084042 (2013)

26. S. Bahamonde, C.G. Böhmer, M. Wright, Phys. Rev. D 92, 104042 (2015)

27. S. Capozziello, V.F. Cardone, H. Farajollahi, A. Ravanpak, Phys. Rev. D 84, 043527 (2011)

28. M. Salti, O. Aydogdu, H. Yanar, F. Binbay, Mod. Phys. Lett. A 32, 1750183 (2017)

29. S. Nojiri, S.D. Odintsov, V.K. Oikonomou, Phys. Rept. 692, 1-104 (2017)

30. Y.F. Cai, S. Capozziello, M. De Laurentis, E.N. Saridakis, Rept. Prog. Phys. 79(10), 106901 (2016)

31. V.K. Oikonomou, Phys. Rev. D 95, 084023 (2017)

32. V.K. Oikonomou, Mod. Phys. Lett. A 32, 1750067 (2017)

33. K. Rezazadeh, K. Karami, P. Karimi, JCAP 1509(09), 053 (2015)

34. R. Herrera, M. Olivares, N. Videla, Int. J. Mod. Phys. D 23(10), 1450080 (2014)
35. M. Jamil, D. Momeni, R. Myrzakulov, Int. J. Theor. Phys. 54(4), $1098(2015)$

36. A. Awad, W. El Hanafy, G.G.L. Nashed, S.D. Odintsov, V.K. Oikonomou, J. Cosmol. Astropart. Phys. 1807(07), 026 (2018)

37. Y.F. Cai, S.H. Chen, J.B. Dent, S. Dutta, E.N. Saridakis, Class. Quant. Gravit. 28, 215011 (2011)

38. H. Abedi, M. Salti, Gen. Rel. Gravit. 47, 93 (2015)

39. K. Izumi, Y.C. Ong, JCAP 1306, 029 (2013)

40. G.G.L. Nashed, W. El Hanafy, Eur. Phys. J. C 74, 3099 (2014)

41. W. El Hanafy, G.L. Nashed, Astrophys. Space Sci. 361(6), 197 (2016)

42. W. El Hanafy, G.G.L. Nashed, Eur. Phys. J. C 75, 279 (2015)

43. K. Karami, A. Abdolmaleki, JCAP 1204, 007 (2012)

44. K. Bamba, R. Myrzakulov, S. Nojiri, S.D. Odintsov, Phys. Rev. D 85, 104036 (2012)

45. M. Salti, O. Aydogdu, I. Acikgoz, Mod. Phys. Lett. A 31, 1650185 (2016)

46. A.I. Keskin, Int. J. Mod. Phys. D 27, 1850078 (2018)

47. A.I. Keskin, I. Acikgoz, Astrophys. Space Sci. 361, 391 (2016)

48. A.I. Keskin, I. Acikgoz, Mod. Phys. Lett. A 32, 1750182 (2017)

49. R. Brandenberger, P. Peter, Found Phys. 47, 797 (2017). https:// doi.org/10.1007/s10701-016-0057-0

50. V.F. Mukhanov, H.A. Feldman, R.H. Brandenberger, Phys. Rept. 215, 203 (1992)

51. E.J. Copeland, M.R. Garousi, M. Sami, S. Tsujikawa, Phys. Rev. D 71, 043003 (2005)

52. G. Calcagni, A.R. Liddle, Phys. Rev. D 74, 043528 (2006)

53. K. Bamba, G.G.L. Nashed, W. El Hanafy, S.K. Ibraheem, Phys. Rev. D 94, 083513 (2016)

54. A.I. Keskin, Int. J. Mod. Phys. D 27, 1850112 (2018). https://doi. org/10.1142/S0218271818501122 